patients with hypertrophic cardiomyopathy. In such cases computed tomography may compete with endomyocardial biopsy in providing a correct diagnosis.

\author{
P Depelchin, ${ }^{\star}$ \\ G Kuhn, $†$ \\ Departments of ${ }^{\star}$ Medical Cardiology and \\ †Roentgenology, \\ Hopital Academique Erasme, Université Libre de \\ Bruxelles, \\ Route de Lennick 808, \\ B 1070 Bruxelles, Belgium.
}

\section{Reference}

1 Siqueira-Filho AG, Cunha CLP, Tajik AJ, Seward JB, Schattenberg TT, Giuliani ER. M-mode and twodimensional echocardiographic features in cardiac amyloidosis. Circulation 1981; 63: 188-96.

This letter was shown to the authors, who reply as follows:
Sir,

The letter by Drs Depelchin and Kuhn documents an additional case of cardiac amyloidosis, in which the computed tomographic appearances are similar to those in our paper (1984; 51: 519-22). They also correctly point out that the description of a postcontrast computed tomogram was omitted in the legend of Fig. $2 b$ in our report.

I would totally agree with their opinion that computed tomography provides important information for the diagnosis of cardiac amyloidosis but that echocardiography still remains the imaging method of choice in the screening of the disease and in the differentiation of the disease from constrictive pericarditis. Computed tomography would, however, be more useful when echocardiography is technically difficult in patients with obesity or emphysema.

Tohru Sekiya,

Department of Radiology,

Jikei University School of Medicine,

Nishi-Shinbashi,

Minato-Ku,

Tokyo 105, Japan.

\title{
Use of the exercise maximal ST segment/heart rate slope in assessing the results of coronary angioplasty
}

Sir,

We have read with interest the article of Silverton et al (1984; 51: 379-85), which essentially reflects our own opinion, except for a detail which we would like to point out. In fact, we are not convinced that the use of bipolar leads, especially CM5, as proposed by the authors, improves the sensitivity of the test dramatically.

We have recorded routine exercise electrocardiograms in patients before and after transluminal coronary angioplasty. All patients underwent bicycle exercise in the sitting position with monitoring leads V2, V5, and aVF. The signals were digitised and processed for averaging and artefact reduction.

In 40 patients who successfully underwent percutaneous transluminal coronary angioplasty the exercise electrocardiogram correlated well with the angiographic results: mean reduction in the degree of stenosis of $72.4 \%$ (range 84.9 to $12.5 \% ; p<0.005$ ), improvement of maximum predicted heart rate $(65.5$ (8)\% to $82(8.6) \% ; p<0.01)$, mean exercise time $(4.8$ $(1.8)$ min to $5.8(1.4)$ min; $\mathrm{p}<0.05)$, maximal ergometric load (133 W/2.8 $\mathrm{min}(18 / 1.2)$ to $152 \mathrm{~W} / 3$ $\min (11 / 1.6) ; p<0.05)$, and maximal rate pressure product $(16.592(128)$ to $25.807(161) ; \mathrm{p}<0.001)$. The maximal ST depression at postangiography ergometry fell from $2.8(1.1) \mathrm{mm}$ to $0.38(0.06) \mathrm{mm}(\mathrm{p}<0.01)$. In 10 patients in whom transluminal coronary angioplasty was unsuccessful we failed to observe any significant change in the exercise electrocardiogram after operation.

While we agree with Silverton et al that exercise testing is of value in the follow up of patients after transluminal coronary angioplasty, we consider that the same good correlations between stenosis reduction and improvement of the exercise electrocardiogram can be obtained with unipolar conventional (V2, V5, aVF) leads.

Leo Finci, Jean Jacques Goy, Raymond Maendly, Milan Grbic, Ulrich Sigwart, Division of Cardiology, Department of Internal Medicine, Centre Hospitalier Universitaire Vaudois, CH-1011 Lausanne, Switzerland. 